INPLASY

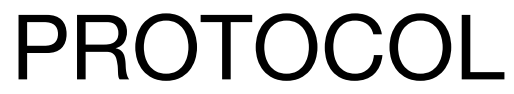

To cite: Moraschini et al. Effectiveness of autogenous graft substitutes for gingival phenotype modification on dental implants: a network meta-analysis. Inplasy protocol 2020100056. doi:

10.37766/inplasy2020.10.0056

\section{Effectiveness of autogenous graft substitutes for gingival phenotype modification on dental implants: a network meta-analysis}

Moraschini, V1; Calasans-Maia, MD²; Shibli, JA3; Sartoretto, SC44.

Review question / Objective: What is the effectiveness of autogenous tissue substitutes to increase KM in peri-implant soft tissues?

Condition being studied: Gingival phenotype modification around dental implants.

Information sources: PubMed/MEDLINE, the Cochrane Central Register of Controlled Trials, Scopus, Embase, and Lilacs. Also, the grey literature was searched.

INPLASY registration number: This protocol was registered with the International Platform of Registered Systematic Review and Meta-Analysis Protocols (INPLASY) on 16 October 2020 and was last updated on 16 October 2020 (registration number INPLASY2020100056).

Review Stage at time of this submission: Risk of bias assessment.

Conflicts of interest:

The authors declare that they have no conflict of interest in the elaboration of this study.

\section{INTRODUCTION}

Review question / Objective: What is the effectiveness of autogenous tissue substitutes to increase KM in peri-implant soft tissues?
Rationale: A wide band of keratinized tissue around dental implants is health related.

Condition being studied: Gingival phenotype modification around dental implants 


\section{METHODS}

Search strategy: PubMed/MEDLINE, the Cochrane Central Register of Controlled Trials, Scopus, Embase, and Lilacs were used to search for articles that were published before September 2020 without other restrictions regarding date or language. A search of the gray literature using the Literature Report and OpenGrey databases was also conducted. Finally, the study reference lists were evaluated (crossreferenced) to identify other studies for potential inclusion.

Participant or population: Patients with dental implants.

Intervention: Autogenous substitutes grafts.

Comparator: Autogenous grafts.

Study designs to be included: Randomized clinical trials.

Eligibility criteria: Patients undergoing treatment for gingival phenotype modification around dental implants with a follow-up period ranging from 6 to 12 months.

Information sources: PubMed/MEDLINE, the Cochrane Central Register of Controlled Trials, Scopus, Embase, and Lilacs. Also, the grey literature was searched.

Main outcome(s): Analyze the variation of keratinized mucosa width (primary outcome) and gingival thickness and participants's aesthetic satisfaction (secondary outcomes) between the baseline and the final follow-up.

Quality assessment / Risk of bias analysis: The RoB 2 (a revised Cochrane risk-of-bias tool for randomized trials) was used to analyze the risk of bias in RCTs. Each study was analyzed in relation to five domains: risk of bias arising from the randomization process, risk of bias due to deviations from the intended interventions, missing outcome data, risk of bias in the measurement of the outcome, and risk of bias in the selection of the reported research. Studies were classified as having a low risk, some concerns, or high risks of bias for each domain. The overall risk of biased judgment used the following criteria: low risk, when the five areas of the study were judged as low risk; some concerns, when the study is judged as raising some concerns in at least one area; and high risk, when the study is judged to be at high risk in at least one domain or when the study is judged to have some concerns for multiple domains in a way that substantially lowers confidence in the result.

Strategy of data synthesis: First, a traditional pairwise meta-analysis was performed. The random-effect model was utilized, incorporating the assumption that different studies evaluated differently, but had related treatment effects. The continuous variables (KMW, GT and PAS) of the included studies were categorized in groups and analyzed using the Review Manager software (version 5.2.8, Copenhagen, Denmark, 2014). The estimates of the intervention effects were expressed as mean differences (MDs) with 95\% confidence intervals (CIs). Chisquared tests evaluated the heterogeneity, which was considered to be low for values $\leq 25 \%$, moderate for values $>25$ but $\leq 50 \%$, and high for values $>50 \%$. Second, a random effect network using Bayesianframework Markov-chain Monte Carlo methods was created using ADDIS 1.16 (https://gemtc.drugis.org). The continuous data of each parameter (KMW, GT and PAS) were evaluated in a network specifying the relationship between the MDs of the studies and combining direct and indirect comparisons of the different types of grafts. The data were considered statistically significant when $P<0.05$, with a $95 \% \mathrm{Cl}$. The probability of the best clinical effect for each type of graft was assessed by calculating the MD of each graft group, compared to arbitrary standard controls and counting the proportion of iterations of the Markov chain of the MD ranking for treatments. 
Subgroup analysis: There will be no subgroup analysis.

Sensibility analysis: To investigate sensitivity, studies classified as having a high risk of bias were excluded.

Language: No language restriction.

Country(ies) involved: Brazil, United States of America, Switzerland.

Keywords: Gingival phenotype modification; autogenous graft; biomaterials; dental implant; keratinized mucosa.

Contributions of each author:

Author 1 - Vittorio Moraschini Conceptualization, study design, data collection, data curation, and writing original draft.

Author 2 - Monica Calasans-Maia - Data collection, data curation, validations, and writing original draft.

Author 3 - Jamil Shibli - Data collection, data curation, validations, and writing original draft.

Author 4 - Suelen Sartoretto - Statistics and writing - review \& editing. 